FORMATION Formation emploi

Revue française de sciences sociales

141 | Janvier-Mars 2018

Quand le tutorat questionne le travail et son analyse

\title{
Les apprentis, au cœur de « l'introuvable relation » formateur-tuteur
}

When the apprentice gets caught in the impossible relation between training teachers and tutors

Auszubildende in der "unauffindbaren Ausbilder-Tutor-Beziehung“

Los aprendices en el centro de la "inhallable relación" formador-tutor

\section{Anne-lise Ulmann}

\section{(Q) OpenEdition}

Journals

Édition électronique

URL : http://journals.openedition.org/formationemploi/5274

DOI : 10.4000/formationemploi.5274

ISSN : 2107-0946

Éditeur

La Documentation française

Édition imprimée

Date de publication : 15 avril 2018

Pagination : 11-26

ISSN : 0759-6340

Référence électronique

Anne-lise Ulmann, « Les apprentis, au cœur de « l'introuvable relation » formateur-tuteur », Formation emploi [En ligne], 141 | Janvier-Mars 2018, mis en ligne le 15 avril 2018, consulté le 30 octobre 2020. URL : http://journals.openedition.org/formationemploi/5274 ; DOI : https://doi.org/10.4000/ formationemploi.5274 


\title{
Les apprentis au cœur de «l'introuvable relation » formateur-tuteur
}

\begin{abstract}
AnNe-Lise UlmanN
Maître de conférences, habilitée à diriger des recherches au CNAM (Conservatoire national des arts et métiers) et membre du laboratoire CRTD (Centre de recherches sur le travail et le

développement)
\end{abstract}

Résumé

Les apprentis au cœur de «I'introuvable relation » formateur-tuteur

À partir d'une observation ethnographique d'un dispositif en alternance, cet article étudie la coopération entre formation et milieu de travail (celui des crèches et des écoles maternelles) visant à préparer de futurs professionnels à exercer leurs fonctions. L'objectif est, d'une part, de montrer que la formation ne peut se réduire à la maîtrise d'actes techniques dissociés des dimensions éthiques et politiques du travail, même pour des métiers peu qualifiés ; d'autre part, de révéler l'importance des conditions d'organisation de l'alternance pour renforcer son potentiel formateur.

Mots clés : apprentissage, formation en alternance, stage de formation, relation écoleentreprise, enseignement sante, formateur, tutorat, puéricultrice, organisation du travail, savoir professionnel, activité professionnelle, processus d'apprentissage, certification, analyse du travail, ethnographie

Abstract

When the apprentice gets caught in the impossible relation between training teachers and tutors

Through an ethnographic observation of an apprenticeship program, this paper examines the cooperation modes established between schools and workplace (nurseries and kindergartens) in order to prepare future professionals to fulfill their functions. In this paper, we seek to show that training cannot be reduced to the mastery of technical acts separated from ethical and political dimensions of work, even for low-skilled occupations. We also intend to reveal the importance of creating organizational conditions that would support apprenticeship program's formative potential.

Keywords: apprenticeship, sandwich training, traineeship, school-enterprise relationship, education by the health ministry, trainer, tutoring, paediatric nurse, work organisation, professional knowledge, vocational activity, learning process, certification, work analysis, ethnography

Journal of Economic Literature: M 53 ; J 24

Traduction : Auteure 
Les formations en alternance se fondent sur l'idée que la confrontation avec les réalités du travail est essentielle pour compléter la transmission des connaissances opérée par l'école, le centre de formation ou l'université. Ce passage par l'expérience d'un travail effectif est d'autant plus souhaité que le développement d'une conception de la compétence ${ }^{1}$, qui nécessite l'effectivité d'une pratique, s'avère également indispensable pour se faire valoir sur le marché de l'emploi.

Le principe de l'alternance, sous la forme d'un contrat d'apprentissage ou d'un stage, désigné désormais par l'expression "période de formation en milieu professionnel " (PFMP) ${ }^{2}$, entraîne des modalités de formation dissociées en deux lieux, l'espace de la formation et l'espace productif. Au sein de ces espaces, formateurs et tuteurs ont la responsabilité conjointe de contribuer au développement des compétences de professionnels en devenir.

Ces tandems tuteur/formateur, considérés comme une "solution salvatrice et un signe d'entente mutuelle entre les milieux éducatifs et ceux des autres activités professionnelles" (ChampyRemoussenard, 2015, p. 22), laissent supposer que leurs modalités de coopération relèvent d'un allant de soi susceptible de mettre fin à une représentation décalée, hors sol, de la formation.

À y regarder de plus près, chaque espace, le lieu professionnel et le lieu de la formation, étant porteurs d'enjeux qui ne sont pas systématiquement complémentaires, la coopération est souvent plus complexe et délicate à instaurer qu'il n'y paraît de prime abord. Ces difficultés peuvent se manifester, à l'extrême, par une sorte de scission tacite entre les deux espaces, réduisant les temps en situation réelle de travail à de simples temps d'emploi, entrecoupés d'absences pour partir en formation.

Cette conception divisée de la formation occasionne souvent des recherches sur la fonction de tuteurs. Il s'agit notamment de révéler comment ils accompagnent les futurs professionnels dans la réalisation du travail, à partir et dans le cours des situations qu'ils ont à traiter (Fillietaz et al, 2014 ; Munoz et Boivin, 2016; Vinatier, 2016). Découvrant, dans un même mouvement, à la fois les dimensions "insues» (Ulmann, 2012) de leur activité et les complexités de leur transmission, ces tuteurs sont fréquemment initiés à l'analyse de leur propre activité comme moyen de développement, pour eux-mêmes comme pour les personnes qu'ils forment (Olry, 2016). Ces analyses, très éclairantes pour expliciter les savoirs et les habiletés ${ }^{3}$ mis en œuvre dans le cours de l'action, tendent à occulter la manière dont sont

1. Les définitions de la compétence sont nombreuses et parfois très différentes (Leplat, 1998 ; Zarifian, 1999, etc.), mais renvoient toutes à la nécessité d'une pratique effective. L'avènement de la compétence en formation nécessite, au-delà des capacités, de prêter attention aux pratiques de travail, donc de pénétrer les mondes professionnels.

2. BO : Circulaire n 2016-055 du 29-3-2016.

3. Le savoir a généralement été appris ou transmis, les habiletés renvoient aux manières d'agir qui prennent en compte les caractéristiques particulières des situations, les savoirs d'action se constituent généralement à partir de récurrences constatées ou éprouvées dans le cours du travail et contribuent à orienter les manières 
organisés, sur un plan pédagogique, les liens avec l'espace de formation. Ce terme désignera indifféremment les CFA (centres de formation d'apprentis), les centres de formation ou les lycées professionnels. L'organisation de cette complémentarité ne relève-t-elle que d'un agencement de procédures? Est-elle sans incidence sur le futur professionnel ?

La manière dont s'articulent l'espace de production et celui de la formation mérite attention et révèle l'importance " des jeux polémiques de ces deux lieux formateurs " (Vincent, 1983) dans l'apprentissage du travail.

En effet, hypothétiquement, les modalités de cette articulation, loin d'être seulement techniques ou procédurales, relèvent non seulement d'une épistémologie de la formation qui ne se réduit pas à la seule acquisition de savoirs, mais viennent aussi interroger, dans le cours même de l'apprentissage, des conflits de légitimité entre le savoir et l'action. La focale d'analyse se centrera donc sur la manière dont chaque espace se mobilise et se coordonne pour développer les compétences attendues des futurs professionnels, ceux-ci se trouvant pris, dans le cours de leur apprentissage, dans les rets de cette "introuvable relation $"{ }^{4}$ formateur-tuteur.

Ce regard sur la formation répond à une double finalité. D'une part, montrer que l'apprentissage d'un métier n'est pas réductible à la maîtrise d'actes techniques dissociés des dimensions éthiques et politiques inhérentes à tout travail, même pour des métiers peu qualifiés. D'autre part, prêter attention aux conditions sociales et environnementales des dispositifs d'alternance pour susciter et développer l'intérêt du travail chez de futurs professionnels.

À cet effet, nous mobilisons une recherche effectuée auprès de futures professionnelles ${ }^{5} \mathrm{de}$ la petite enfance passant le CAP (certificat d'aptitude professionnelle PE - petite enfance) ou le diplôme d'auxiliaire de puériculture (AP). Ces deux formations présentent l'intérêt d'articuler aux enjeux de l'apprentissage de l'action ceux de la certification et de la reconnaissance sociale d'un travail dévalorisé.

Après la présentation succincte de ces deux certifications relevant du champ de la petite enfance, il conviendra d'identifier et de comprendre les différentes sources de légitimation de l'action. Seront abordés, notamment, le rapport au savoir pour les formateurs et la partition théorie/pratique. Nous aborderons ensuite l'analyse des différentes configurations de tutorat et la manière dont se maillent, au sein de la structure d'accueil (association, structure publique ou privée), l'organisation du travail et celle du tutorat. Enfin, seront étudiées les deux modalités d'évaluation permettant de certifier les compétences acquises dans le cadre de ces dispositifs de formation construits selon un principe d'alternance.

d'agir comme de penser le travail. Dans le travail, les trois se trouvent fréquemment mobilisés conjointement. 4. C'est en référence à l'ouvrage dirigé, en 1986, par Lucie Tanguy, "Lintrouvable relation formation/emploi ", que nous qualifions ainsi la relation tuteur/formateur.

5. Nous emploierons désormais le féminin, tant les femmes sont majoritaires dans ces métiers. 
La conclusion reviendra sur les enjeux d'un apprentissage professionnel qui déploie une pensée sur le travail, sans se polariser sur les seuls gestes professionnels.

Sur un plan théorique, cette recherche s'inscrit dans une perspective interdisciplinaire et compréhensive. Le travail n'est pas réductible à l'emploi, mais, en référence aux cliniques du travail, il y est pensé comme une praxis où les sujets ne sont pas seulement agis par les injonctions et les procédures, mais ont une part active dans les situations qu'ils rencontrent.

\section{Encadré 1. Méthodologie}

Cette recherche, effectuée à la demande de la Caisse nationale d'allocations familiales (CNAF), portait sur la qualité de l'accueil et la socialisation des enfants avant cinq ans.

À partir d'une double démarche de type ethnographique, réalisée pendant dix-huit mois dans les structures d'accueil comme dans l'espace de formation, nous avons analysé la manière dont les professionnelles travaillaient et étaient formées par les formatrices et les tutrices. Cette approche a permis de saisir le travail en train de se faire, dans l'espace de formation, en suivant les situations d'apprentissage préparées par les formatrices. Dans l'espace professionnel (crèche, halte-garderie, école maternelle), nous avons cherché à comprendre comment étaient mobilisés les apprentissages de la formation dans les situations réelles de travail.

Pour deux apprenties, nous avons suivi la trajectoire d'apprentissage complète, de la formation à la structure d'accueil (des crèches). Pour les autres élèves de ces lycées professionnels ou des CFA (centres de formation d'apprentis), pour lesquels le suivi complet ne s'est pas avéré possible, nous nous sommes plus particulièrement appuyés sur le suivi partiel qu'effectuaient les formatrices. À cet effet, nous les avons accompagnées lors de l'observation des retours de stages, des visites dans la structure d'accueil et des modalités d'évaluation.

Ces observations, à quelques exceptions, n'ont pas été filmées, mais notées à la manière des ethnographes. A intervalles réguliers (tous les trois mois), elles ont été retravaillées avec les professionnelles concernées, notamment lors de leurs réunions où nous étions témoin-interlocuteur (Ulmann, 2012). Nos observations ont donc fait l'objet d'échanges avec les équipes sur leur travail et notre façon de le voir.

Du côté de la formation, nous avons mobilisé la même méthodologie de travail, en observant plus particulièrement les enseignements professionnels, les visites dans les structures d'accueil, les retours de stages et les évaluations en situation de travail. Ces observations ont été complétées par des entretiens et de nombreux échanges portant plus largement sur leurs conceptions du travail et leurs trajectoires professionnelles.

Pour l'ensemble des formations observées, nous avons rencontré, à trois reprises, les stagiaires qui l'acceptaient, lors d'entretiens individuels portant plutôt sur leurs choix d'orientation, leur intérêt pour le travail et à la fin de la formation, sur leurs projets professionnels.

Dans la continuité de cette approche, la conception de la formation développée ici rompt avec une approche fonctionnaliste qui réduit les apprentissages à des usages immédiats, sans comprendre ce qui les fonde. En référence aux travaux plus récents d'une psychosociologie du travail (Lhuillier et al., 2013), qui prolonge les apports des psychosociologues par ceux des ergonomes et des cliniciens du travail, il convient de considérer qu'un acte 
formatif ne peut relever de la seule conformation. Il se trouve en effet toujours à l'articulation de trois champs : celui de la vie psychique (la personne), celui des conduites humaines (le groupe) et celui du champ social (l'institution) (Enriquez, 1992). L'acte de travail destiné à permettre les adaptations, les ajustements et les transformations à des évolutions sociales et professionnelles suppose donc des choix. Les personnes ont en effet à arbitrer entre plusieurs déterminants conscients et inconscients, notamment les prescriptions, les règles, leurs valeurs, leurs habitus sociaux et culturels, les points de vue de leurs collègues... Pour la formation, cette conception de l'homme au travail implique des modes d'accompagnement et d'apprentissage qui ne clivent pas la personne entre ces déterminants, entre sa vie au travail et sa vie hors travail, mais l'aident tout au contraire à consolider son unité sociale et professionnelle.

\section{Quelles sources de légitimation de l'action?}

Après une brève présentation du contexte des formations considérées, cette partie explore les tensions éprouvées par les futures professionnelles entre l'abondance des normes à prendre en compte dans le travail et les règles apprises en formation.

\subsection{La certification, un enjeu pour la reconnaissance professionnelle}

Les métiers auprès des enfants, comme la plupart des métiers du care, pâtissent d'un manque de reconnaissance qui a plusieurs causes. D'abord, la fréquente association aux registres domestique, parental et féminin qui en masque les dimensions professionnelles ; puis, l'orientation vers ces fonctions de jeunes filles faiblement scolarisées ; enfin, la disjonction entre, d'un côté, l'emploi, davantage reconnu, même s'il est faiblement rémunéré, et de l'autre, le travail, qui est fait quotidiennement. Celui-ci, quand il est regardé, est enjolivé, parce que les bébés, dans notre imaginaire social, séduisent davantage que le travail administratif ou industriel (Ulmann, Rodriguez et al., 2015). Cet attrait pour les petits estompe le réel de l'activité. Il maintient dans l'invisibilité les compétences déployées pour faire face à la complexité des missions, en tension entre une relation de care (prendre soin des enfants) et une relation de service (répondre aux demandes des parents, souvent exigeants s'agissant de leur progéniture) (Delgoulet et al., 2011 ; Ulmann, 2012).

Le CAP petite enfance (PE) et le diplôme d'auxiliaire de puériculture $(\mathrm{AP})^{6}$ sont les deux qualifications les moins élevées de ce champ. Bien que de niveau équivalent, niveau $\mathrm{V}^{7}$,

6. Ces deux diplômes relèvent tous deux d'un niveau V. Ils ne dépendent pas du même ministère de tutelle, ministère de la Santé pour les AP et Éducation nationale pour les CAP-PE. Pour plus de précisions sur ces différences, voir Ulmann, Rodriguez, Guyon, 2015.

7. Niveau de formation équivalent au CAP (certificat d'aptitude professionnelle), au BEP (brevet d'études 
soit le plus bas dans la nomenclature des diplômes au regard du marché de l'emploi, leur accès à la formation diffère. Le CAP PE est en effet ouvert à tous sans conditions d'entrée ; le diplôme d'AP suppose quant à lui l'obtention d'un concours qui exclut les personnes faibles dans les matières générales et tend à attirer des jeunes femmes surdiplômées en recherche d'emploi. Pour les deux diplômes, une partie des épreuves dites "pratiques" s'effectue en co-évaluation avec les professionnelles du métier, soit directement sur le lieu du travail, soit par le biais de cas reconstitués.

Revaloriser les faibles qualifications de ces métiers, attachés au féminin et qui ont tendance à s'ethniciser, est une tâche délicate, à laquelle s'attèlent les formatrices, souvent d'anciennes puéricultrices ${ }^{8}$ à l'hôpital, devenues directrices de crèche avant de choisir la voie de la formation.

Pour contrer ces représentations, les formatrices ont développé deux stratégies :

- La première étaie le travail sur le savoir médical pour mieux le faire valoir ;

- La seconde développe une conception de la professionnalité épurée d'influences non légitimes, comme l'éducation ou la culture, pour distendre pratiques sociales versus parental et pratiques de travail versus professionnel : "Les bisous ou les petits noms aux enfants, c'est à la maison! Ici, vous êtes des professionnelles... ", entend-on régulièrement dans les enseignements des établissements étudiés.

\subsection{Forces et insuffisances de l'apprentissage expérientiel}

L'alternance est une modalité pédagogique ancienne qui confronte de futurs professionnels à des situations réelles de travail. De type expérientiel, cet apprentissage a une valeur formative qui s'explique doublement. D'une part, il permet d'apprendre ce qui ne peut s'apprendre sans un passage par une pratique, le fameux "learning by doing" (apprendre en faisant) ; d'autre part, il offre la possibilité d'agir à un débutant, et ce faisant, il contribue à construire "sa propre expérience du monde à travers un processus d'essai et d'erreur" (Geay, 1991, p. 1).

Cependant, ce qui s'apprend par cette confrontation directe aux situations est forcément lié à des contraintes locales, situées, qui ne permettent pas une montée en généralité dans d'autres cas de figure. L'expérience en tant que telle est nécessaire, mais reste lacunaire en matière de compréhension plus distanciée des mobiles d'une action, qui permettrait de les mobiliser dans d'autres situations. Lui fait défaut ce que Pastré (2011) nomme, à partir des travaux de Vergnaud (1996), la mise à jour d'une "organisation invariante " susceptible de structurer des repères pour d'autres "classes de situations". La

professionnelles) ou au diplôme national du brevet (DNB, et anciennement brevet des collèges ou BEPC). 8. Pour le CAP PE, les formatrices sont plutôt d'anciennes éducatrices de jeunes enfants, ce qui renforce les clivages entre ces deux diplômes. Concernant les éléments que nous développons ici sur l'hygiène, les points de vue sont partagés entre les formatrices des deux diplômes. 
formation s'avère donc nécessaire pour compléter ce manque, ce qui redonne place au "Learning by teaching" (apprendre par l'enseignement).

La manière d'occuper cette place renvoie cependant à des conceptions très différentes de l'acte de former. En effet, les uns vont plutôt dissocier les savoirs des apports de l'expérience ; les autres, au contraire, chercheront à articuler les dimensions pratiques et conceptuelles d'une activité professionnelle. Pour les premiers, les tenants de la dissociation, le travail de montée en généralité s'effectue par des apports complémentaires, qualifiés de "théoriques ». Pour les seconds, il ne s'agit pas d'apports théoriques, mais de théoriser à partir de cette pratique, pour extraire la conceptualisation qui l'organise. "Au fond de l'action, il y a une conceptualisation" rappelle Vergnaud (1996).

Cherchant d'abord à revaloriser le travail, avant de le faire apprendre, les formatrices rencontrées estiment plutôt nécessaire "de leur faire apprendre la théorie qu'elles ne trouveront jamais dans les structures ${ }^{9}$. Le rapport entre le travail effectué dans les structures d'accueil et celui évoqué lors des « séquences charnières » que sont les retours en formation, est alors dissocié et revendiqué comme tel.

Ce choix est aussi lié aux représentations que les formatrices ont de ces publics. La formation doit, d'une part, "enraciner un éthos particulier " (...) ; d'autre part, instruire " (Boltanski, 1969, p. 25) La place de l'expérience compte donc moins que le respect et l'apprentissage de savoirs.

\subsection{Le primat de l'autorité rationnelle}

Les savoirs, notamment ceux liés à l'hygiène, s'imposent comme des références intangibles, alors même que les jeunes filles, fortes de leurs expériences dans les structures professionnelles, tentent de faire valoir d'autres points de vue :

"-Vous dites qu'on doit laver les matelas de change à chaque fois, mais ça, madame, c'est pas vrai! Dans ma crèche, on ne le fait jamais et ma tutrice m'a dit que ce n'était pas la peine, que d'abord on ne peut pas, on n'a pas le temps!"

- Comment ça, ça ne se fait jamais ?! Et sil a une infection? Vous trouvez ça bien pour les autres?

- Ah oui... non mais là... De toute façon, dans ma crèche, quand ils sont malades, on ne les prend pas... " (Retour de stage 2, AP, CFA : infirmière, formatrice à l'école d'infirmières, vacataire dans le centre de formation pour les auxiliaires de puériculture).

Se référant au domaine médical, l'apprentissage des règles s'impose et ne se discute pas, même si les futures professionnelles marquent, comme c'est le cas ici, leur étonne-

9. Formatrice AP. CFA, techniques éducatives (infirmière puéricultrice, directrice de crèche, puis formatrice depuis plus de cinq ans). 
ment. Pour cette formatrice, ancienne infirmière, les références au savoir médical sont les seules sources légitimes de l'action. L'échange se clôt assez vite, alors même qu’à ses débuts, d'autres jeunes femmes manifestaient les mêmes préoccupations.

L'apprentissage de la règle détachée de son usage permet ici de substituer "une autorité rationnelle fondée sur un savoir à l'autorité traditionnelle de la famille ou du milieu ". (Durkheim, 1928, p. 22). Cette autorité rationnelle ne se fonde pas sur la pratique ou l'expérience, mais sur un savoir "positif» qui se transmet par le livre et l'école (Boltanski, Ibid). L'imposition de cette " autorité rationnelle » est cohérente avec l'idée d'une professionnalité sans affect, dont la fonction sert à se différencier de la pratique profane. Cet apprentissage de principes scientifiques énoncés comme règles d'action et appropriés progressivement comme une morale de travail, revient à la formation. Il s'agit de préparer le travail en stage, comme si ce savoir, même sans usage, était requis, telle une toile de fond d'une culture professionnelle.

Cet apprentissage désincarné des règles installe une distance avec l'activité de travail. La légitimité de l'action est alors clairement du côté d'un savoir considéré comme scientifique, même si le travail d'appropriation didactique finit par en écorner la scientificité, pour devenir ce que Boltanski (op. cit.) nomme "des savoirs en miettes». Réduits au champ scolaire, ces savoirs, coupés de leur fondement, conforment à un mode de pensée pour préparer au travail professionnel. Pour les formatrices, le lien à l'action n'est pas absent. Cependant, il ne se situe pas du côté de l'activité de travail, mais du côté des rapports de travail pour apprendre à y tenir sa place.

\subsection{Des normes de travail et des manières d'agir plurielles}

Outre les étonnements à propos de règles qui ne servent pas, les difficultés d'apprentissage tiennent également à la diversité des normes de travail, impliquant des "débats de normes » (Schwartz, 1998 ; 2012) :

E., stagiaire AP en retour de stage, évoque une situation qu' elle qualifie de "trop dure " et paraît très émue. Elle s'occupe du repas d'un petit groupe d'enfants de deux ans, et l'un d'eux refuse tous les plats. Elle essaie alors de le faire manger en lui racontant une histoire, mais sa tutrice la reprend sévèrement, au motif qu'elle outrepasse son rôle. Une telle attitude reviendrait aux parents, et non à une professionnelle.

Les « séquences charnières " entre la structure d'accueil et la formation sont fréquemment saturées de telles situations où « l'action qui convient " dépend d'un très grand nombre de paramètres, difficiles à identifier en formation simplement à partir des récits où se lisent les épreuves subjectives du travail. Cette abondance de normes entre en tension avec l'imposition d'un savoir médical qui souffre peu la prise en compte d'autres références. Les futures professionnelles semblent alors prises, au sein même de la formation, entre plusieurs logiques. D'une part, une logique de soumission à un savoir maître qui contribue à valoriser le travail ; d'autre part, une logique d'adaptation aux diverses exigences du lieu 
professionnel, sans pouvoir réellement mettre en débat les buts visés par ces exigences et les manières de s'y prendre. D’un côté, le savoir médical s’impose, mais de l'autre, les règles de travail de la structure obligent également.

Que faire apprendre si les alternatives et les débats de normes échappent à la formation ? Comment penser l'apprentissage pour éviter d'être éprouvé ? "Pour moi, c'est juste impossible de laisser pleurer un tout petit pour ne pas l'habituer aux bras... » expliquera une auxiliaire qui décide d'arrêter la formation. La perception de ces complexités et leur absence d'analyse ne contribuent-elles pas à rendre le travail aussi éprouvant, voire à développer des pratiques clandestines ? Ne se joue-t-il pas ici, dans ce temps d'apprentissage, des enjeux importants de santé au travail?

\section{Le tutorat, une modalité formative aux contours flous}

Le terme de tutorat recouvre de nombreuses pratiques, dont aucune n'est clairement définie. S'il s'agit souvent de former ou d'accompagner une personne débutante, les attendus de la fonction sont flous. Qu'attend-on du tuteur en matière d'apprentissages pour ces débutantes : " montrer ", " expliquer ", " apprendre à faire ", " déléguer », " partager "... le travail ? Les réponses à ces questions varient d'une structure à une autre. Elles donnent lieu à des modalités d'apprentissage qui vont du simple accueil d'une personne, sans participation, même "périphérique " au travail (Lave, Wenger, 1991), à une participation complète relevant quasiment de la tenue du poste de travail.

La manière de prendre en charge les apprenties, limitée à un cadrage juridico-administratif, ajustée au fonctionnement de la structure ou envisagée comme s'il s'agissait de recevoir une collègue potentielle, donne à voir des apprentissages de nature très différente.

\subsection{Dissoudre la pédagogie dans un cadrage juridico-administratif}

L'articulation entre les deux lieux est une préoccupation qui se traduit fréquemment par un cadrage administratif foisonnant (conventions, cahiers de liaison, fiche navette...). Ces artefacts médiatisent techniquement la relation aux structures d'accueil, en qualifiant l'alternance de juxtapositive ou d'intégrative (Labruyère, Simon, 2014).

Réduite à l'administratif, l'articulation pédagogique est parfois reportée sur les apprenties qui doivent, avant le départ en stage, écrire dans leur livret "leurs objectifs d'apprentissage " pour la période considérée : "J'avais marqué comme objectif que je voulais faire des transmissions aux parents, mais ça n'a jamais été possible» (AP, retour de stage 1, CFA, 20 ans).

Peut-on demander à celui qui apprend de savoir ce qu'il doit apprendre ? Ce report de la responsabilité formative sur son destinataire n'est-il pas l'indice d'un "impensé pédago- 
gique» (Maubant, 1997) qui masque le questionnement sur les usages pédagogiques des tensions inhérentes à ces deux lieux ? Comment ces modalités administratives, intégratives ou juxtapositives engendrent-elles des changements dans le rapport au savoir ou à l'action?

\subsection{L'accueil des apprenties reflète le fonctionnement interne}

L’observation de l'accueil des apprenties dans les écoles maternelles a fréquemment montré qu'elles arrivaient le matin sans savoir ce qu'elles feraient dans la journée. Si elles n’ignoraient pas l'organisation globale d'une journée, très cadrée par des tâches récurrentes (horaires des repas, nettoyage des locaux...), elles n'en savaient guère plus et se trouvaient là sans projection possible sur la journée complète. Passive en apparence, mais non intérieurement, cette attente permet d'apprécier silencieusement si le partage du travail avec la tutrice est équitable: "J'ai compris... Chaque fois qu'elle peut me coller au ménage pendant qu'elle va dans les classes, elle n'hésite pas!" (CAP, Lycée pro 17 ans).

Lacceptation, par la stagiaire, de cette position périphérique ou vicariante tient par la qualité de la relation avec la tutrice. Quand elle peut s'installer, cette relation de proximité est une occasion d'échanges indirects sur le travail qui en découvrent "les principes d'action", sans toujours passer par un "principe d'explication" (Delbos et Jorion, 1987). Cela facilite la conduite intégrale d'actions qui permet, en retour, de valoriser le travail : "C'est moi qui ai organisé l'atelier dessin avec les petits de la garderie " (CAP, Lycée pro, 17 ans). A contrario, quand il s'agit de réaliser uniquement des tâches partielles, la période de formation en milieu professionnel (PFMP) est une épreuve et les tensions sont fréquentes avec les tutrices.

\subsection{De l'apprentie à la collègue potentielle}

Le tutorat peut être envisagé de manière plus collective. Dans ce cas, la personne débutante n'est pas confiée à une personne pour l'accompagner dans son apprentissage, mais plutôt intégrée à une équipe.

Cette forme de tutorat suppose une organisation collective qui n'est pas seulement liée à l'accueil d'une nouvelle professionnelle. Dans ce cas de figure, les apprentissages du tutorat rompent avec le principe de transmission de la "bonne pratique " qui existerait en soi, pour ouvrir sur les arbitrages de travail réalisés par chacun dans les situations rencontrées. Cette forme de tutorat ouvre sur un empan temporel plus large, comme si l'intégration dans le métier et les valeurs qui le traversent ne se dissociaient pas de son apprentissage.

E., apprentie auxiliaire, change une petite fille de 20 mois, à côté de G., auxiliaire expérimentée dans la même équipe de travail, qui change un bébé de quelques mois. E. porte la petite fille pour la poser sur la table à langer. G., tout en changeant le bébé, la regarde rapidement. Elle la laisse commencer son travail puis lui dit :

"- G: Tu ne devrais pas la porter pour la mettre sur la table. A force, si tu fais ça, tu ne vas pas tenir.. le dos en prend un coup... 
- E. : Oui je sais, mais là, comme on est un peu à la bourre, ça va plus vite.

- G: Oui, mais c'est quand on va vite justement. Tu sais, ils sont malins, sills voient que tu portes, ils ne vont pas se presser non plus... Je dis ça, c'est pour toi, pour que ... le dos après...

- E. : Ouais, mais t'as raison parce que le soir..."

Dans ces échanges, ce que G. semble faire apprendre à E. n'est pas tant la manière de s'y prendre pour faire monter à l'enfant les quelques marches, qu'une manière de penser son action dans une temporalité plus lointaine que celle de l'instant présent. À cette prise en compte d'une temporalité moins immédiate, elle insère un savoir très élaboré sur les enfants, dont les comportements seraient en interaction avec ceux des professionnelles.

L'apprentie, prise par les exigences du moment, se décale progressivement de l'action en cours, et apprend indirectement à reconsidérer la hiérarchie des buts de son activité : d'abord se protéger, puis respecter les contraintes de temps et non pas l'inverse. Dans cet exemple, G. parle à une collègue potentielle du travail dans sa globalité et non d'une manière dépliée qui procéderait, tel le référentiel, par domaine : les règles, le savoir à propos des muscles dorsaux, les procédures...

Le travail évoqué dans ces quelques échanges est celui qui est éprouvé dans sa complexité, sans dissociation du corps et de la tête, sans rupture entre la vie dans le travail et la vie en dehors.

Ces présentations rapides des manières de penser et d'agir le tutorat, en lien avec l'espace de formation, tendent à confirmer l'hypothèse selon laquelle les conditions d'accueil d'une professionnelle en devenir sont déterminantes pour le développement de ses compétences. On constate que dans les fonctionnements où prévaut le partage collectif des questions du travail, l'intégration des apprenties est moins pesante pour les tuteurs. Qu'il s'effectue dans une relation duale ou de manière collective, l'espace d'accueil ouvre des points de vue nouveaux sur le travail qui le désenclavent de représentations trop rigides.

\section{La certification à l'épreuve du travail réel}

En prêtant attention aux manières d'évaluer les compétences, puisque l'alternance vise aussi la certification, s'impose le constat que les modalités d'évaluation maintiennent une dimension scolaire, encore loin d'une " alternance dialogique " (Denoyel, 1999).

\subsection{Le comportement comme substitut à l'attention prêtée au travail}

Concernant le CAP-PE, les évaluations s'effectuent sur le lieu de stage, mais à distance du travail. Lévaluation - dans le bureau de la directrice et avec la formatrice et la tutrice - du 
stage de $M .^{10}$, une jeune-fille connue dans le lycée professionnel pour être indisciplinée et souvent absente des cours, l'illustre bien :

"Formatrice: "Je viens faire le point avec vous sur le stage de M. Comment ça se passe?

Directrice : "Ecoutez, c'est formidable. je crois que tout se passe à merveille »

Formatrice "Je suis ravie d'entendre cela, parce que ce n'est pas toujours ce que l'on me dit sur M.» (...)

Directrice : "Non, elle fait exactement ce qu'elle $e^{11}$ lui dit, obéis parfaitement, ne prend pas d'initiatives qui ne vont pas, vraiment c'est parfait...C'est l'Atsem (Agent territorial de service en école maternelle) parfaite... Elle est calme, posée, demande toujours l'autorisation avant de faire quelque chose avec les enfants " (...)

Formatrice, en s'adressant à $M:$ "Et pourquoi en cours alors, on ne peut pas toujours en dire autant?"

Pour ces fonctions souvent considérées comme subalternes au regard de l'apprentissage du savoir, même à l'école maternelle, l'attention sur ce qui est fait concrètement est rarement à l'ordre du jour dans les entretiens.

La directrice "croit que tout" se passe bien, sans se questionner plus avant sur la réalité de cette impression et sans savoir aussi ce que recouvre précisément ce " tout». La formatrice, de son côté, ne questionne pas plus la tutrice, présente et silencieuse, comme si l'essentiel était le comportement professionnel qui se subsumerait par la mise en visibilité d'attitudes respectueuses d'un certain ordre social, évaluées par la directrice. Dans la grille d'évaluation de compétences remplie à la fin de l'entretien, les items " autonomie ", "ponctualité", "application et soin" auront les meilleures cotations, ceux sur " compréhension des consignes" et "rapidité dans l'exécution" seront un peu moins bien notées, pour "laisser une marge de progrès» dira la formatrice, illustrant parfaitement que les compétences repérées « dépendent de la manière de les voir " (Stroobants, 2009, p. 179)

Lévaluation dans l'espace professionnel garde une configuration scolaire par le cadre institué, mais elle n'aborde pourtant ni les savoirs liés aux tâches professionnelles, ni même ceux enseignés dans les cours. S'instaure alors une béance entre les discours visant à revaloriser ces professions et l'absence d'attention prêtée au travail réel.

En revanche, la place réservée au respect des formes du travail et le temps qui y est consacré, au cours de l'entretien, questionnent le sens de ces attendus. Le comportement fait-il alors office d'artefact destiné à combler les ignorances du travail réellement attendu ? Ce regard sur le comportement, indépendamment du travail à produire, ne conduit-il pas à le rabattre

10. M. a 18 ans. Elle a fait l'objet d'un renvoi à la suite d'un conseil de discipline pour insolence à l'égard de la directrice de son lycée.

11. La directrice fait allusion à une enseignante avec qui M. a un peu travaillé. 
sur des qualités allant à l'encontre des efforts faits, dans les discours, pour le revaloriser ? Lévaluation sert-elle à s'assurer d'une bonne conduite ou d'un travail bien fait ? Ce peu d'attention accordée au travail effectué ne serait-il pas symptomatique de la reconnaissance sociale de ces métiers?

\section{2 À la recherche du savoir dans le faire}

Pour les auxiliaires de puériculture, l'évaluation procède à l'inverse, s'attachant à observer, à partir d'une activité courante dans la vie de la crèche, les manières d'agir de la professionnelle débutante. L'activité évaluée est une tâche habituelle (un jeu, un repas...) qui se déroule sous les regards de la formatrice, de la tutrice et parfois de la directrice. Celles-ci observent pendant vingt à trente minutes les manières d'agir de l'apprentie en essayant de ne pas trop perturber les enfants, toujours un peu impressionnés par ces regards insolites.

À l'issue de cet exercice, la formatrice, la plupart du temps en présence de la tutrice, conduit un entretien dit de "débriefing " de cette activité, où il est attendu une analyse réflexive sur l'activité réalisée. Il est alors demandé à la jeune femme de dire comment, de son point de vue, s'est passée l'activité. Cette question, qui semble laisser ouverte la possibilité d'exprimer ses affects après l'épreuve, s'avère être, au contraire, le commencement d'un deuxième exercice redoutable, conduisant souvent aux pleurs, où le savoir s'impose de manière absolue.

La formatrice ouvre un questionnement très serré sur les raisons de ce qui s'est passé lors de la conduite de l'activité. À ce questionnement, les réponses de l'apprentie sont alors renvoyées à un "oui mais..., précisez..., dites pourquoi... » de la formatrice. Ainsi, si l'activité de peinture s'est bien passée, la stagiaire doit en trouver les raisons, non pas dans une réflexion sur ce qui a été pensé lors de la préparation, mais dans les savoirs qu' elle aurait appris en formation. Pour la peinture, la bonne réponse doit faire référence aux cours sur la psychologie qui précisent des âges sur la motricité fine des enfants ou sur leur capacité à comprendre le partage des objets... Très fréquemment, les réponses finissent par être apportées par la formatrice qui clôt l'exercice par "vous ne connaissez pas vos cours» ou "vous êtes trop dans le faire et pas assez dans le savoir-faire».

Cet exercice, également éprouvant pour la tutrice qui, parfois, s'excuse de ne pas avoir su développer suffisamment les pratiques réflexives chez la jeune fille, révèle que les actes professionnels ne sont jamais vus pour ce qu'ils sont, mais pour ce qu'ils supposent de savoirs incorporés.

Il ne semble pas admis qu'ils puissent être faits sans référence à ces savoirs, parce qu'ils seraient alors intuitifs. Cette épreuve tend à déposséder les savoirs de la pratique de leur richesse et de leur complexité, pour les ramener à des contenus scolaires ayant une validité absolue parce qu'ils sont extraits de disciplines scientifiques. J. Friedrich (2014), reprenant les analyses de Ryle, montre pourtant que ce qui se donne à voir ne dépend pas d'une supposée intériorité, même s'il ne s'agit pas de nier qu'une « compréhension intellectuelle des règles et des prescriptions (...) est attestable dans nos pratiques quotidiennes. (...) Le savoir-faire, 
cette intelligence impliquée dans la pratique, n’est pas réductible au savoir et possède une spécificité» (p. 177). Ce dispositif d'évaluation révèle que la formation paraît tenir pour quantité négligeable cette intelligence de la pratique. Ces modalités évaluatives interrogent sur la place laissée aux savoir-faire qui, pour être attestés comme scientifiques, ne doivent pas être intuitifs ou proches de pratiques domestiques.

La tutrice, dans ce moment particulier de l'évaluation, se place toujours en retrait de la formatrice, comme si cette dernière était la garante, pour l'ensemble de la profession, de la revalorisation de ces métiers par les savoirs. La reconnaissance sociale impose-t-elle de cliver savoir et savoir-faire ? Le savoir-faire doit-il être relégué à la pratique clandestine ? De telles méthodes ne vont-elles pas à l'encontre de la reconnaissance justement recherchée ? Enfin, ces clivages ne peuvent-ils pas, à force, ruiner la santé ?

\section{Conclusion}

Chercher à éclairer les complexités de l'articulation des deux lieux formateurs de l'alternance, celui de la formation et celui de l'activité professionnelle, montre, à partir d'une observation attentive du travail dans ces deux espaces, que leur articulation dans la construction pédagogique relève davantage d'une réflexion épistémologique sur le rapport aux savoirs et à leurs usages dans l'action que d'un cadrage juridico-administratif, censé organiser la complexité de cette articulation.

Apparaissent ainsi deux logiques différentes, engendrant des conflits de légitimité dans les processus d'apprentissage mis en ouvre par les formateurs et les tuteurs. Les premiers, ceux de l'espace de formation, se situent dans une logique rationnelle " qui est aussi celle de la raison linéaire" (Delbos et Jorion, p. 34) ; les seconds les abordent dans leur globalité puisque coexistent et s'hybrident des données de l'environnement social et institutionnel, des histoires de vie, des pratiques culturelles, des savoirs, «l'ensemble des connaissances disponibles se cristallisant dans leur totalité en niveau d'intégration successif» (Ibid).

Pour un professionnel en devenir, cette double situation offre l'opportunité d'une exploration de ces différentes logiques relevant de ce que nous définissons comme s'enseigner du pluriel et construire progressivement sa singularité en développant une "pensée émancipatrice» (Rancière, 2004). Tenter d'aligner les processus d'apprentissage sur l'une ou l'autre de ces logiques serait vain et pourrait conduire à dévitaliser le potentiel formateur de ce dispositif pédagogique.

Au-delà de l'alternance et du domaine de la petite enfance, dont les caractéristiques liées au domestique et au féminin ne sont pas à sous-estimer, ces principes de travail invitent à interroger la manière dont est pensée, depuis la formation, la construction d'une professionnalité pour contribuer à la reconnaissance d'un métier.

Fonder la légitimité de l'action professionnelle à partir d'un déni des habitus culturels ne peut-il pas conduire à une pratique désincarnée ? N'est-il pas risqué, pour la santé, de désarticuler ce qui fait lien entre ce que l'on est et ce que l'on fait, entre ce qui fait sens dans le 
travail et hors travail ? Doit-on, au nom de la professionnalisation, appauvrir le travail de ses dimensions culturelles?

\section{Bibliographie}

Boltanski L. (1969) "Prime éducation et morale de classe ", Cahier du centre de sociologie européenne, V, Paris, École pratique des hautes études, Sorbonne, Mouton.

Champy-Remoussenard P. (2015), "Les transformations des relations entre travail, éducation, formation dans l'organisation sociale contemporaine : questions posées par trois dispositifs analyseurs ", Revue française de pédagogie, n 190, pp. 15-28.

Delbos G., Jorion P. (1984/1990), La transmission des savoirs, Paris, Maison des Sciences de l'Homme.

Delgoulet C., Weill-Fassina A., Mardon C. (2011), « Pénibilités des activités de service et santé des Agents Spécialisés des Écoles Maternelles. Des évolutions avec l'âge " @ctivité, revue électroniqque, $\mathrm{n}^{\circ} 1$, pp. 2-25.

Denoyel N. (1999), "Alternance tripolaire et raison expérientielle à la lumière de la sémiotique de Peirce ", Revue Française de pédagogie, n 128-1 pp. 35-42.

Durkheim E. (1928/2013), Education et sociologie Paris, PUF, coll. «Quadrige».

Enriquez E. (1992), L'organisation en analyse, Paris, PUF.

Fillietaz L., Remery V., Trebert D. (2014), «Relation tutorale et configurations de participation à l'interaction. Analyse de l'accompagnement des stagiaires dans le champ de la petite enfance "in @ctivité, vol. 11-1,pp. 22-46.

Friedrich J. (2014), "Le savoir-faire : un savoir ou autre chose ? " in Friedrich J., Pita Castro J.-C., Recherches en formation des adultes. Un dialogue entre concepts et réalité, Dijon, Editions Rimes et Passions.

Geay A. (1991), "L'alternance éducative ", Actualité de la formation permanente, n 115.

Labruyère C., Simon V. (2014), "L'alternance intégrative, de la théorie à la pratique ", Bref-Céreq, n 328.

Lave J., Wenger E. (1991/2003), Situated learning. Legitimate peripheral participation, Cambridge University press.

Leplat J. (2013), «Les gestes dans l'activité en situation de travail. Aperçu de quelques problèmes d'analyse ", Perspectives interdisciplinaires sur le travail et la santé, $\mathrm{n}^{\circ} 15$.

Lhuillier D., Andrade de Barros V., Garcia de Araujo J.-N. (Dir) (2013), « La psychosociologie du travail. Perspectives internationales ", Nouvelle Revue de Psychosociologie $\mathrm{n}^{\circ} 15$, Toulouse, Erès. 
Maubant P. (1997), " Pour une didactique et des pédagogies de l'alternance », Revue Pour, $\mathrm{n}^{\circ} 154$, pp. 141-161.

Munoz G., Boivin S. (2016), "Accompagner les tuteurs à expliciter leur connaissanceen-acte : l'usage de l'instruction au sosie en formation en soins infirmiers, Education Permanente, $\mathrm{n}^{\circ}$ 206, pp. 109-117.

Olry P. (2016), "Tutorat en formation initiale professionnelle et transmission du métier. Le cas d'élèves sage-femmes ", Education Permanente, n 206, pp. 29-46.

Pastré P. (2011), La didactique professionnelle. Approche anthropologique du développement chez les adultes, Paris, PUF.

Rancière J. (2004), Le maître ignorant. Cinq leçons sur l'émancipation intellectuelle, Paris, 10/18.

Ryle G. (1949/1978), La notion d'esprit. Pour une critique de concepts mentaux, Paris, Payot.

Schwartz Y. (1988/2012), Expérience et connaissance du travail, Paris, Editions sociales.

Stroobants M. (2009) «Dénouer les ficelles de métier pour connecter les savoirs formels et informels ", Techniques et culture, n' 51, pp. 164-179.

Tanguy L. (1986), L'introuvable relation formation-emploi. Un état des recherches en France, Paris, La Documentation française.

Ulmann A.-L. (2012), Le contrôle : traçage et transmission. Regards sur l'activité des contrôleurs des caisses d'allocations familiales, Sarrebruck, Éditions universitaires européennes.

Ulmann A.L. (2012), "Le travail émotionnel des professionnelles de la petite enfance ", Revue des politiques sociales et familiales, $\mathrm{n}^{\circ} 109$, pp. 47-57.

Ulmann A.-L., Rodriguez D., Guyon M. (2015), « Former les futures professionnels de la petite enfance : entre soin et éducation, quelle place pour les affects? ", Revue des politiques sociales et familiales, $\mathrm{n}^{\circ} 120$, pp. 31-43.

Vergnaud G. (1996), "Au fond de l'action la conceptualisation », in Barbier J.-M, Savoirs théoriques et savoirs d'action, Paris, PUF, pp. 275-292.

Vinatier I. (2016), "Le conseil pédagogique : une activité en tensions ", Education Permanente, $\mathrm{n}^{\circ} 206$.

Vincent F. (1983), Les tuteurs en entreprises, Centre d'éducation permanente, Paris, Publications de la Sorbonne.

Zarifian P. (1999, Objectif compétence, Paris, Editions Liaisons Sociales. 